\title{
Techniques for generating centimetric drops in microgravity and application to cavitation studies
}

\author{
Philippe Kobel · Danail Obreschkow • \\ Aurèle de Bosset $\cdot$ Nicolas Dorsaz $\cdot$ Mohamed Farhat
}

Received: 15 April 2008/Revised: 20 December 2008/Accepted: 5 January 2009/Published online: 1 March 2009

(C) The Author(s) 2009. This article is published with open access at Springerlink.com

\begin{abstract}
This paper describes the techniques and physical parameters used to produce stable centimetric water drops in microgravity, and to study single cavitation bubbles inside such drops (Parabolic Flight Campaigns, European Space Agency ESA). While the main scientific results have been presented in a previous paper, we shall herein provide the necessary technical background, with potential applications to other experiments. First, we present an original method to produce and capture large stable drops in microgravity. This technique succeeded in generating quasi-spherical water drops with volumes up to $8 \mathrm{ml}$, despite the residual g-jitter. We find that the equilibrium of the drops is essentially dictated by the ratio between the drop volume and the contact surface used to capture the drop, and formulate a simple stability criterion. In a second part, we present a setup for creating and studying single cavitation bubbles inside those drops.
\end{abstract}

P. Kobel $(\bowtie) \cdot$ D. Obreschkow - A. de Bosset · N. Dorsaz .

M. Farhat

Laboratoire des Machines Hydrauliques,

EPFL, 1007 Lausanne, Switzerland

e-mail: philippe.kobel@a3.epfl.ch

P. Kobel

Max-Planck Institut für Sonnensystemforschung,

Max-Planck-Straße 2, 37191 Katlenburg-Lindau, Germany

D. Obreschkow

Department of Astrophysics,

University of Oxford,

Keble Road, Oxford OX1 3RH, UK

N. Dorsaz

Institut Romand de Recherche Numérique

en Physique des Matériaux, EPFL,

1015 Lausanne, Switzerland
In addition, we analyze the influence of the bubble size and position on the drop behaviour after collapse, i.e., jets and surface perturbations.

\section{Scientific background}

A large spherical liquid body in absence of gravitational forces is a paradigmatic system to study a variety of physical phenomena. Yet the control of its position and stability in a residual acceleration environment is a delicate task. Acoustic positioning techniques, developed for the drop physics module (DPM, flown onboard space shuttle Columbia mission STS-73), overcame this problem while allowing the manipulation of perfectly isolated centimeter-sized levitating drops (described in detail by Croonquist 1993). This complex technique has proved fruitful for dynamical studies such as the equilibrium shapes of rotating drops (Wang et al. 1986), or the rheological properties of oscillating drops with adsorbed surfactants (Apfel et al. 1997; Holt et al. 1997; Chen et al. 1998). However, many applications do not require the direct manipulation of free-floating drops, and can be successfully achieved with a captured drop. Particular instances are the study of free surface phenomena, e.g., Faraday waves (Faraday 1831; Holt and Trinh 1996), thermocapillary flows (Treuner et al. 1995), or liquid jets (Lord Rayleigh 1879). For these purposes, a much simpler system could be used, such as an injector tube able to attach and stabilize the drop. We designed and implemented such a drop generation system, and used the ensuing centimetric drops as nearly isolated spherical volumes in which single cavitation bubbles may be investigated. 
The behaviour of cavitation bubbles-depressurized vapor bubbles appearing naturally in many industrial systems - has been demonstrated to be strongly dependent on the liquid geometry and the nature of nearby surfaces, as both impose boundary conditions on the pressure field of the fluid (as reviewed in Brennen 1995). In particular, these geometrical conditions influence the violent emission of liquid jets and shock waves by collapsing bubbles, identified as the main sources of cavitation erosion in hydraulic machinery (Isselin et al. 1998; Shima 1997). Therefore recent fundamental studies focussed on the dynamics of bubbles and associated phenomena next to flat (Brujan et al. 2002) and curved (Tomita et al. 2002) rigid surfaces, or flat free surfaces (Robinson et al. 2001; Pearson et al. 2004). However, on-ground investigations inside static water flasks are restricted to probe flat free surfaces due to gravity, and the water volume can hardly be isolated. To extend these possibilities, we used a microgravity environment to produce spherical water drops, inside which we studied cavitation bubbles. It should be emphasized that our experiment did not aim at investigating the direct effects of microgravity on cavitation, but used microgravity as a way to produce a spherical water drop of large size, which is crucial to vary physical parameters such as the size and position of the bubble within the drop (see Sect. 3.4). Moreover, this drop geometry presents unique properties influencing the bubble dynamics: a spherical, closed free surface and an isolated volume.

Our experiment was flown on parabolic flights, offering sequences of microgravity phases lasting for about 20 s (ESA 42nd microgravity research campaign 2006, 8th student parabolic flight campaign 2005). A variety of scientific outcomes has been presented by Obreschkow et al. (2006), including (1) double-jet formation, (2) shock wave-induced secondary cavitation and (3) shorter collapse time than in extended water volumes.

This paper aims at presenting in detail the instrumentation designed for the creation of the drop and the generation of the bubble, as well as the physical parameters influencing the performance of the experiment. It is structured in two main parts: the first part (Sect. 2) addresses the generation of a centimetric drop and its stability in the residual g-jitter, while the second part (Sect. 3) describes the setup used to create and observe cavitation bubbles inside the drop. The latter section also includes an analysis of the drop state (liquid jets emission, drop stability) after bubble collapse with respect to the bubble position and size. To conclude, a brief summary is given in Sect. 4, including some prospects of the drop generation system.

\section{Generation of centimetric drop in microgravity}

\subsection{Drop generation system}

During each microgravity phase (20 s duration), one captured centimetric drop was created as follows. Distilled water was smoothly expelled through a custom-designed Aluminium injector tube (Fig. 1a) by a step-motor micropump (APT Instruments SP200 peristaltic pump). The injector tube was filled with hydrophilic porous foam for two purposes. First, to guarantee a homogeneous laminar flow and thereby control the actual drop volume during its formation. Second, to favor the attachment of the drop by water capillary cohesive forces through the tube foam interface. In addition, the top edge of the tube was trimmed at an angle of $45^{\circ}$ in order to increase the effective contact surface with the drop. This inclined edge was coated with hydrophilic aluminium oxyde $\mathrm{Al}_{2} \mathrm{O}_{3}$ to ensure efficient contact, while the tube exterior was coated with hydrophobic silicon to prevent the water from flowing down. Four injector tubes with outer diameter $D=16,12,9$ and $7 \mathrm{~mm}$ have been used (see Fig. 1a). They were interchanged according to the water drop volume to be produced, as larger contact surfaces allow to stabilize larger volumes (cf Sect. 3).

The micropump was programmed by an external computer, which triggered the pumping start, controlled its flux and its duration. The micropump was automatically started whenever the gravity level dropped below $0.05 \mathrm{~g}$ for more than $1 \mathrm{~s}$ (see Sect. 3.1). The flux was set to $0.6 \mathrm{ml} / \mathrm{s}$ and reduced to $0.3 \mathrm{ml} / \mathrm{s}$ during the $2-3$ last seconds of the pumping process. This flux reduction aimed at slowing down the drop motion and avoiding subsequent drop oscillations. Varying the pumping time allowed to adjust the drop size. To precisely control the drop volume, the tube was filled with water up to its top foam interface prior

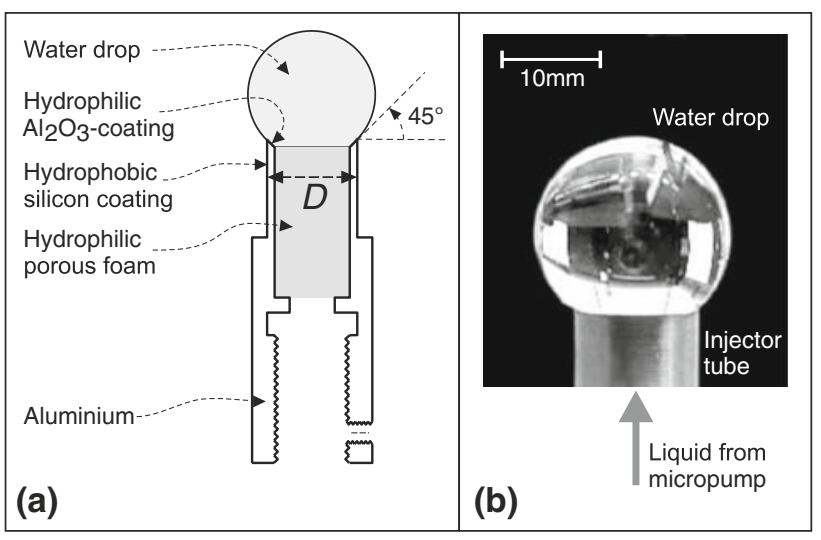

Fig. 1 a Vertical cross section of the injector tube. b Quasi-spherical drop in microgravity 
to start the pumping. Thus, drop volumes of $2.4-8 \mathrm{ml}$ (diameters of 16-25 mm) were formed in 5-15 s, respectively (the maximal pumping time was purposely restricted to $15 \mathrm{~s}$ according to Sect. 2.2). After each microgravity phase, the water drop fell down under hypergravity and was passively absorbed by sponges covering the vessel bottom.

\subsection{Drop oscillations in g-level jitter}

During the microgravity phases provided by parabolic flights, the residual g-jitter can reach values of the order of $0.02 \mathrm{~g}$ at typical frequencies of $1-10 \mathrm{~Hz}$. In $10 \%$ of the zero-g parabolas however, we recorded flight-based deviations from the microgravity level larger than $0.02 \mathrm{~g}$ for durations longer than $0.2 \mathrm{~s}$. They mostly occurred approximately $3 \mathrm{~s}$ before the end of the zero-g phases. The injector tube being fixed to the aircraft reference, those fluctuations induced observable multi-pole oscillations of the water drop, visible in Fig. 2. Although the g-jitter spectrum contains eigenfrequencies of the drop (see Bauer and Chiba 2004, for a theoretical evaluation of the natural frequencies of captured drops), no resonance was observable and these oscillations always damped out rapidly within characteristic times of $0.5-1 \mathrm{~s}$. We argue that the large area of support attaching the drop to the tube $\left(1.1 \mathrm{~cm}^{2}\right.$ for an outer diameter of $\left.12 \mathrm{~mm}\right)$ was responsible for this efficient attenuation, as the large porous foam ensures an inelastic rebound of the water drop. Due to the strong g-jitter sometimes seen in the final $3 \mathrm{~s}$ of the microgravity phase, we limited the pumping time to $15 \mathrm{~s}$, thus creating the most undisturbed spherical drops for the cavitation studies described in Sect. 3 .
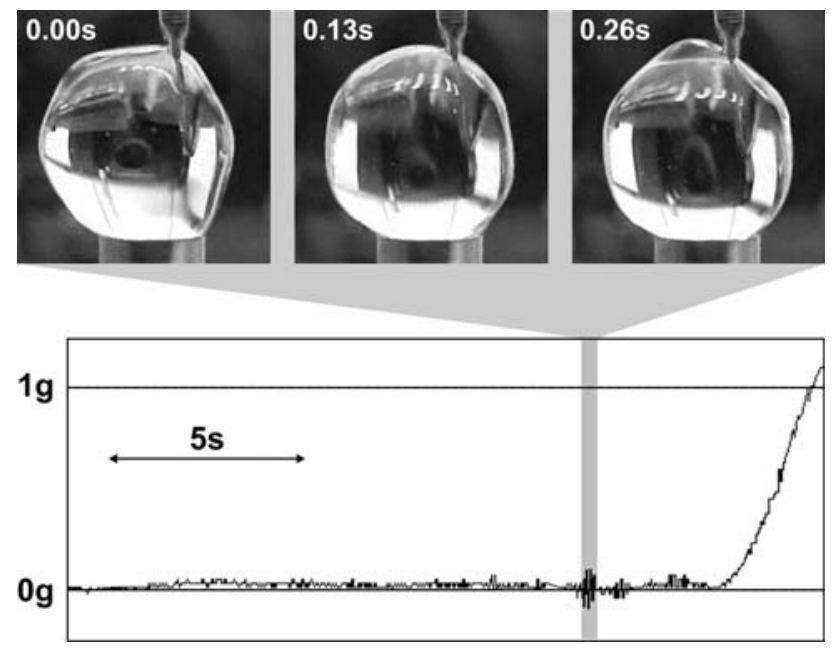

Fig. 2 Top: water drop oscillations resulting from g-jitter superior to $0.05 \mathrm{~g}$, as filmed by the standard video camera (Sect. 3.1). Bottom: vertical component of the acceleration vector versus time
We note that unwanted oscillations could also be partly avoided by increasing the viscosity of the fluid (e.g., using a solution of glycerol/water), or by using a second injector tube stabilizing the top of the drop, as realized by Treuner et al. (1995). On the other hand, the observed multipole oscillations qualitatively compare to the theoretical calculations of a harmonically excited captured drop by Bauer and Chiba (2004), indicating another potential application of this drop generation system.

\subsection{Drop equilibrium on injector tube}

Depending on the drop volume and the injector tube diameter, the drop could be unstable and sometimes even detached from the injector tube before the end of the microgravity phase. This leads to the following question: given a tube diameter, what is the maximum drop volume that is assured to remain attached on the tube under normal unevittable g-jitter?

We answer this question by formulating a simplified stability criterion for the drop, based on its mechanical equilibrium in the tube non-inertial frame. Only the force balance is considered, while the torques and shearinduced drop deformations are neglected. In the frame of the tube, the inertial force acting on the drop volume is opposed to its acceleration, $F_{V}=-\rho V a$, where $V$ is the drop volume, $\rho=1,000 \mathrm{~kg} \mathrm{~m}^{-3}$ its mass density and $a$ the residual acceleration (g-jitter) of the tube in microgravity. In reaction to this volume force, the cohesive forces at the tube interface develop a counterforce $F_{S}=\sigma_{S} S$, where $S$ is the tube surface and $\sigma_{S}$ the surface force density along the direction considered. The drop will be stable as long as the tube surface force compensates the volume force, namely $\rho V a<\sigma_{S \text {, max }} S$, where $\sigma_{S, \text { max }}$ is the maximal value of the surface force density that can be exerted by the tube interface (considered as an intrinsic property of the tube). Although $\sigma_{S, \max }$ is in principle direction-dependent, we treat it as an average over all directions. During a standard parabola, the g-jitter reaches a maximum value $a_{\max }=0.02 \mathrm{~g}$ and the stability criterion can be formulated as:

$\frac{V}{S}<\frac{\sigma_{S, \max }}{\rho a_{\max }} \equiv c$

Since the critical parameter $c$ is a constant, the ratio $V / S$ dictates whether the drop remains stable during the microgravity phase.

This theoretical result could be verified in our microgravity experiment, which also allowed a phenomenologial determination of $c$ as follows. For all 155 parabolas (summing over both flight campaigns), we collected the drop volumes $V$ and tube surface areas $S$ and represented them as pairs $(S, V)$ in the diagram of Fig. 3. In total, 


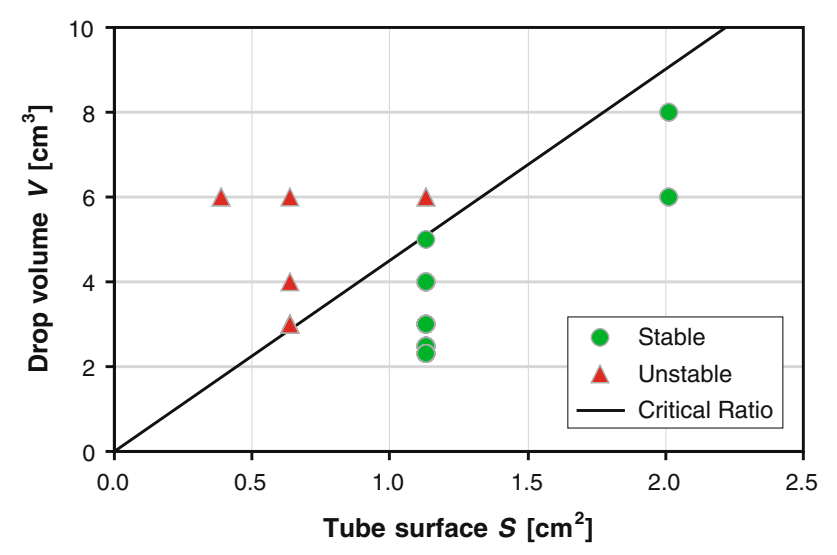

Fig. 3 Diagram representing the different pairs $(V, S)$ of drop volume $V$ and tube contact surface $S$ used in the course of the experiment

fourteen different $(S, V)$ configurations were probed, corresponding to the fourteen data points. Each of these configurations was repeated in a variable number of parabolas. If during all of these parabolas, the drops remained stable on the tube (i.e., never detached and hanged on the side of the tube), the corresponding point was classified as stable (circles), otherwise as unstable (triangles).

In this representation, the critical parameter $c$ corresponds to the slope of a straight line passing through the origin and separating the unstable from the stable regime; graphically, we find $c \sim 4.5 \mathrm{~cm}$. This value has to be taken as a safe stability limit, as no configuration with $V / S<4.5 \mathrm{~cm}$ was unstable. For the 49 experimental cycles with $V / S>4.5 \mathrm{~cm}$, the average rate of drop detachment was about $25 \%$. Hence, configurations with $V / S>4.5 \mathrm{~cm}$ are not necessarily unstable, as rapid variations of the acceleration vector can prevent large displacements of the drop or even bring it back to a stable state. Therefore the relation (1) should be exclusively taken as a stability criterion, as its violation is only a necessary but not sufficient condition for unstability.

We like to end this analysis with a note for additional physical insight: given the observed value $c \sim 4.5 \mathrm{~cm}$, we can estimate the maximal surface force density $\sigma_{S, \max }$ using (1). One finds $\sigma_{S, \max } \sim 10 \mathrm{~N} \mathrm{~m}^{-2}$, which compares well with the cohesive force of water observed in droplets suspended under their weights (see Young 1845, lecture L for further discussion on that matter).

Stimulated by this reflection, we can find a physical interpretation for the length $c$ by noting that the ratio $V / S$ corresponds to the height of a cylinder with base surface $S$ and volume $V$. Thus $c$ can be seen as the maximally allowed height of a hypothetical water cylinder, such that can be sustained by the tube interface in a weak gravitational field of $0.02 \mathrm{~g}$.

\section{Generation and study of cavitation bubble}

\subsection{General setup}

Figure 4 shows the arrangement of the central components of the setup. The water drop, the injector tube as well as the electrodes for the bubble generation were contained in a sealed transparent cubic vessel $(20 \mathrm{~cm}$ side length, $1 \mathrm{~cm}$ thickness) made of clear high-density polycarbonate (Lexan).

To record the fast bubble dynamics, we used a highspeed CCD camera (Photron Ultima APX, up to 120,000 frames/s) with a framing rate of 12,500-24,000 frames/s (depending on the temporal and spatial resolution desired), and an exposure time of $5 \mu$ s. The record sequence had a duration of $11 \mathrm{~ms}$, during which the required illumination was provided by a perpendicularly placed high-power flashlight (Cordin Light Source Model 359). The slow water drop formation was filmed with a standard video camera (Sony Camcorder DCR-TRV900, 25 frames/s).

A diagram illustrating the links between the different functional units of the experiment as well as the order of their activation in time is given in Fig. 5. A control computer program triggered the micropump (drop creation), the electrodes discharge (bubble generation), the image recording and the flashlight release. The interface between the computer signals (USB) and the manual start button, micropump and electrodes was performed by standard input and output boards. The experimental sequence was automatically initiated at the beginning of each flight parabola, as soon as a stable level of microgravity $(<0.05 \mathrm{~g})$ was reached for more than $1 \mathrm{~s}$. The gravity data was provided by a $100 \mathrm{~Hz}$ accelerometer (Memsic, 2125 Dual-axis Accelerometer) that continuously recorded the gravity level during the whole flight. Immediately after the water drop formation, the electrical discharge for bubble generation was released. Because of the low trigger time accuracy of the discharge generator,

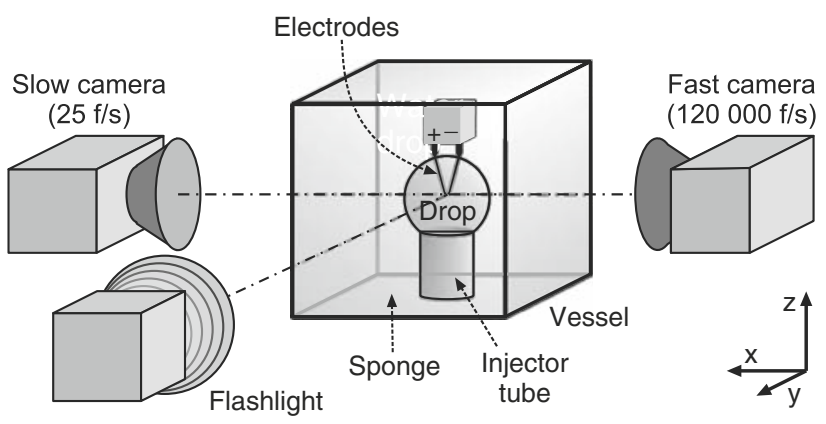

Fig. 4 Configuration of the optical system surrounding the central components 


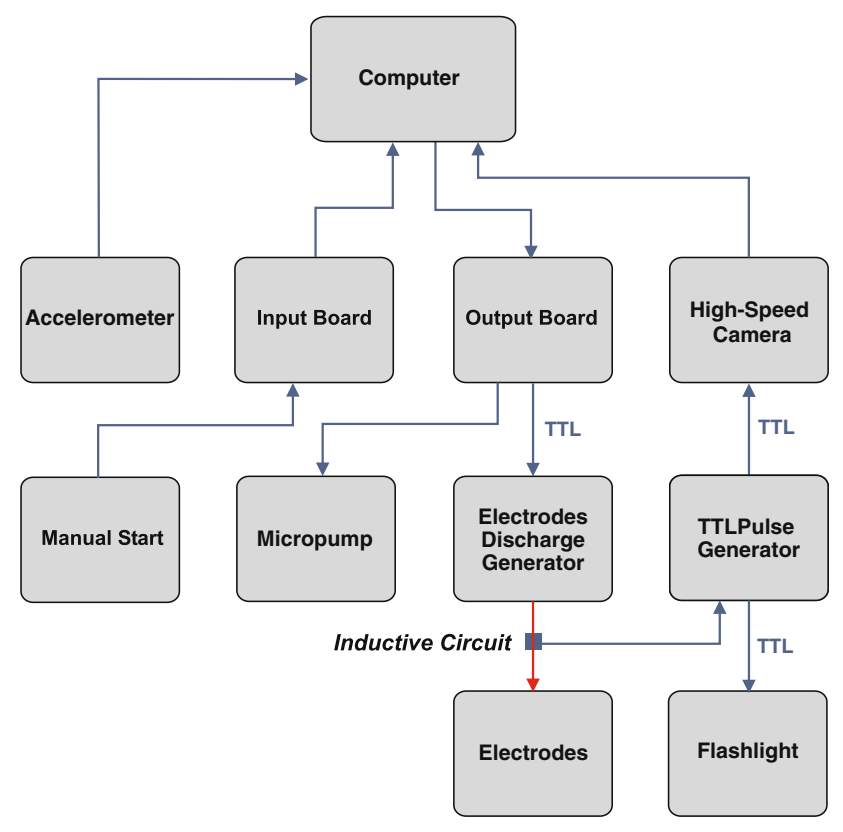

Fig. 5 Flow diagram of the different functional units of the experiment in sequential order

we used the electrodes discharge pulse current to trigger the recording system through an inductive response circuit. This precaution ensured that the recording system started at the instant of bubble creation, in order to capture the entire bubble dynamics and subsequent phenomena. For the exact synchronization of the high-speed camera and the flashlight, the induced signal from the electrodes was split by a TTL pulse generator sending a pulse to both units simultaneously.

The experiment was designed to be redundant in case of failure of any unit: As an alternative for the accelerometer, the experimental sequence could be started manually from an external button or directly from a computer key. If none of these devices were functional, the electrodes discharge generator could still be fired manually instead of being triggered by the computer. Finally, the recording system could be triggered directly from the electrodes inductive signal in case the TTL pulse generator were defective, but with a somewhat less accurate synchronization. However, none of these off-nominal procedures had to be used over the 155 parabolas.

The experimental setup was fixed on a single rack containing horizontal levels, as shown in Fig. 6. The lower level (base plate) was used to attach the experiment on the plane rails and to carry the heavy units (high-power generators for electrodes and flash light, camera control unit), while the upper level (working table) carried the devices requiring in-flight manipulation.

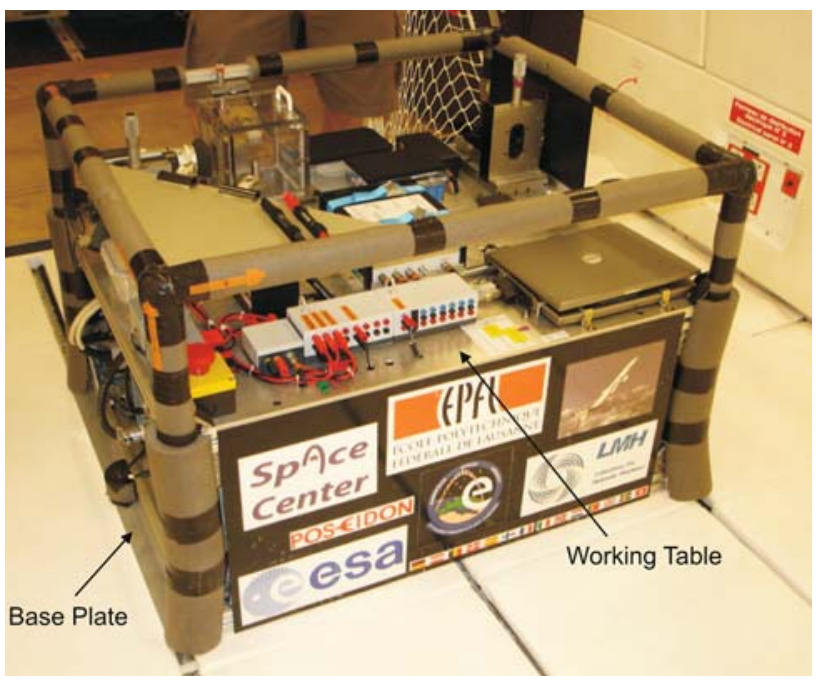

Fig. 6 Picture of the two-level rack carrying the experiment

\subsection{Spark-based technique and design of electrodes}

The cavitation bubble was generated using the standard spark-based technique, by which a spark released by electrodes allows the conversion of electrostatic energy into a point-like plasma of high temperature and pressure. The plasma immediately recombines and gives rise to a thermally growing bubble, which subsequently collapses under the surrounding liquid pressure (Chahine et al. 1995). This standard spark-based technique was chosen instead of a laser-based technique because of the difficulty to focus a laser beam across a (variable) spherical surface, and for aircraft security reasons.

To produce the discharge, a pair of electrodes was immersed in the water drop from above (Fig. 7b-e). The required electrostatic energy was stored into capacitors, whose fast discharge was enabled by a triggered spark gap (see Pereira et al. 1994 for more details). These devices were
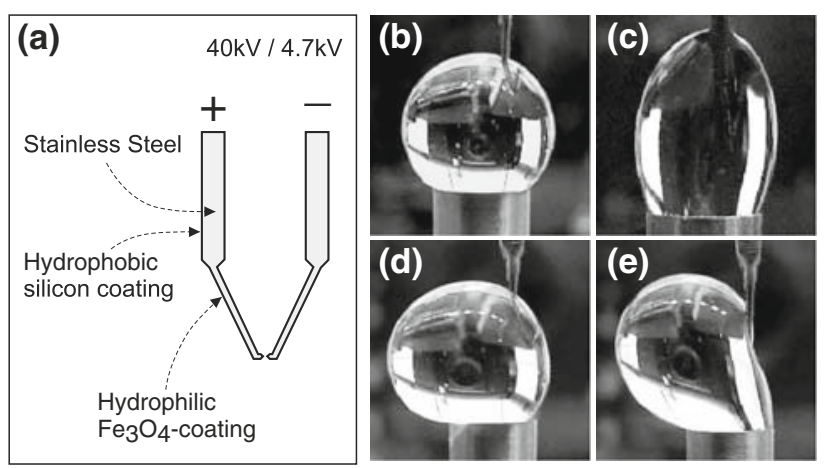

Fig. 7 a Schematics of the electrodes with their coating; b quasispherical drop, $\mathbf{c}$ oblong attracted drop, $\mathbf{d}$ slightly repulsed drop, e strongly repulsed drop 
contained in the unit called electrodes discharge generator in Fig. 5. The capacitor's voltage $(4.7 \mathrm{kV})$ was high enough to induce the breakdown of water molecules and thereby create the plasma between the electrodes. To vary the bubble size, the discharge capacitance could be stepwisely altered between 30, 50, 100 and $200 \mathrm{nF}$. A minimum capacitance value of $30 \mathrm{nF}$ was necessary to ensure water breakdown, probably due to the potential drop occuring during the finite impedance fall-time of the spark gap.

The electrodes had a thicker part which was mounted on an axis controllable by micro-positioners, and thinner ends to penetrate the drop (see Fig. 7a). The latter had a thickness of the order of $0.5 \mathrm{~mm}$ with a spacing between the tips of about $1 \mathrm{~mm}$. Using the positioners, the electrode tips could be displaced in three dimensions, allowing their precise positioning inside the water drop.

In order to minimize the interaction of the electrodes with the water volume, their tips were coated with hydrophilic ferrous oxyde $\mathrm{Fe}_{3} \mathrm{O}_{4}$ and their upper part covered with hydrophobic silicon, as illustrated in Fig. 7a. Despite the careful preparation of the electrodes, we observed significant water drop distortions induced by electrodewater interaction. These distortions were strongly dependent on the specific coating of the electrodes, particularly on the thickness of the silicon layer covering their thick upper part. Without any silicon coating, the upper part of the electrodes was too hydrophilic, yielding an oblong distortion of the water volume (Fig. 7c). Conversely, additional silicon engendered water repulsion by the electrodes (Fig. 7d,e). Because of this effect, the electrodes position had to be readjusted during the flight to control the direction of liquid jets emission following bubble collapse (directed towards to and away from the closest free surface element, see Sect. 3.4), so that they did not escape from the high-speed camera field of view. Moreover, the drop deviations from a perfect sphere hinder the measurement of quantitative parameters such as the curvature ratio between drop and cavity, relative bubble eccentricity $\varepsilon$ and relative bubble radius $\alpha$ (defined in Sect. 3.4). These parameters are nevertheless of importance for the analysis and modeling of the observations. The above thus reveals the necessity to optimize the electrodes surface coating and to render the tips finer in order to minimize such distortions.

\subsection{Discharge-cavity energy relation}

To characterize the efficiency of our spark-based system, we determined the fraction of electrical discharge energy actually transformed into bubble energy. The electrical discharge energy $E_{\mathrm{e}}$ is given by the stored electrostatic energy of the capacitors. The hydrodynamic potential energy of the bubble $E_{\mathrm{b}}$ relates to its maximum radius $R_{\mathrm{b} \text {, max }}$ (as achieved at the end of its growth) via
$E_{\mathrm{b}}=\frac{4 \pi}{3} R_{\mathrm{b}, \max }^{3},\left(p_{\infty}-p_{v}\right)$, where $p_{\infty}$ is the ambient static pressure and $p_{\mathrm{v}}$ the water vapor pressure at room temperature. In order to determine the relation $E_{\mathrm{b}}\left(E_{\mathrm{e}}\right)$ experimentally, we therefore measured the maximum bubble radii $R_{\mathrm{b}, \max }$ for the different values of the capacitance.

To complement the flight measurements within the drops, we also carried out on-ground measurements inside water flasks with additional values of the capacitance: 10 and $20 \mathrm{nF}$. For the measurements inside the drops, we corrected the apparent $R_{\mathrm{b} \text {,max }}$ using a model of optical refraction through a spherical surface (see Appendix). Care was taken to select bubbles sufficiently far from the drop surface to ensure the validity of the refraction model as well as to avoid bubble shape disturbances. For each measured value of $R_{\mathrm{b} \text {, max }}$, the corresponding bubble energy $E_{\mathrm{b}}\left(R_{\mathrm{b}, \max }\right)$ was determined using the relation above, taking into account the different ambient pressures on ground and inside the aircraft cabin, $10^{5}$ and $(8 \pm 0.2) 10^{4} \mathrm{~Pa}$, respectively. Note that the cabin pressure contains an uncertainty owing to dynamic changes in the course of a parabola (e.g., during throttle-back maneuvers), but we did not correct for these changes (between different measurements) here and considered the pressure constant over the bubble lifetime. The mean values of $E_{\mathrm{b}}$ are plotted against $E_{\mathrm{e}}$ in Fig. 8. The errors on $E_{\mathrm{b}}$ are the standard deviations of the $E_{\mathrm{b}}\left(R_{\mathrm{b}, \max }\right)$ measurements, while the $E_{\mathrm{e}}$ errors account for the standard precision of capacitance values of about $5 \%$. A weighted least-square linear regression (accounting for both errors) yielded the following discharge-cavity energy relation: $E_{\mathrm{b}}$ $=0.024( \pm 0.005) E_{\mathrm{e}}$.

The obtained relation reveals that about $2-3 \%$ of the capacitors electrostatic energy was transformed into potential energy of the bubble, whereas the remaining energy was absorbed elsewhere. The latter fraction was presumably dissipated in electrical losses and radiated in the form of a shock wave caused by the initial plasma

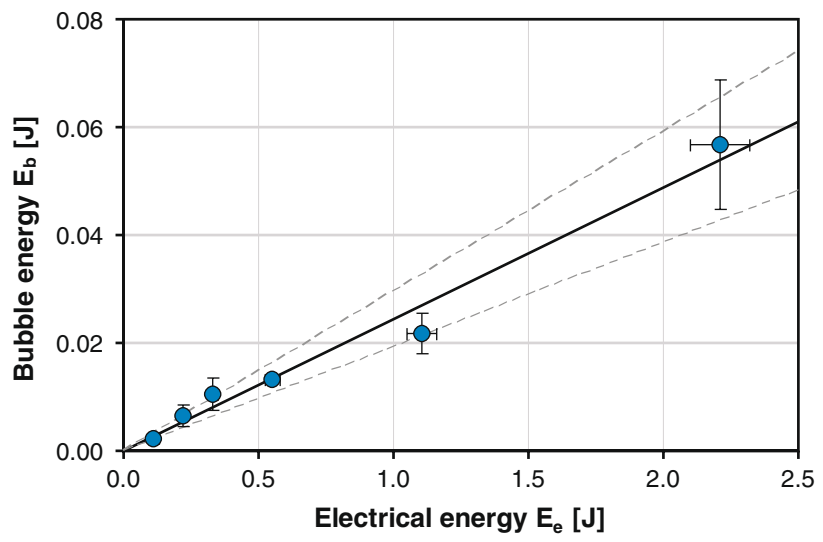

Fig. 8 Mean bubble energy as a function of capacitors electrostatic energy. Solid line: weighted linear regression. Dashed line: regression uncertainties 
expansion (Chahine et al. 1995). To estimate the contribution of the shock wave proper, we used the method described in Obreschkow et al. (2006). Explicitely, we assumed that most of the shock energy was converted into submillimetric bubbles, arising from the excitation of impurities at the multiple passages of the shock confined within the drop. By integrating the potential energies of all these bubbles (using the energy-maximum radius relation given above), we found the fraction of shock wave energy to be roughly $4 \%$ (cf Obreschkow et al. 2006, where it was found that the shock wave energy is about $50-70 \%$ of the sum of the shock wave energy and bubble energy, confusingly referred to as the "initial electrical energy"). By taking into account additional sources of energy loss such as heat production and light emission (Brenner et al. 2002; Leighton 1994), the actual energy deposited in the water by the electrodes can be estimated to be of the order of $10 \%$. The remaining $90 \%$ correspond to the initial energy fraction lost in electrical Joule heating, electro-magnetic radiation, and plausible incomplete discharge of the capacitors (as the electrodes voltage drops below the breakdown threshold of water). Although these values depend much on the specific devices used, they are roughly consistent with previous estimations (Chahine et al. 1995).

\subsection{Liquid jets and drop stability after bubble collapse}

Next to a free surface, a cavitation bubble undergoes a toroidal collapse emitting a high-speed microjet directed away from the local surface element, while a counterjet called splash forms on the free-surface (Blake and Gibson 1981; Robinson et al. 2001; Pearson et al. 2004). In the present drop experiment, two liquid jets were then seen to escape the drop in opposite directions (Obreschkow et al. 2006). We observed that the importance of these liquid jets strongly depends on the bubble size and position within the drop. Furthermore, the collapsing bubble could induce instabilities on the drop surface or even cause a burst of the entire drop, thus rendering the jets investigation impossible. This motivated us to study the influence of the geometrical configuration of the bubble within the drop on these particular phenomena.

To characterize the jets emission and drop stability in function of the system's geometry, we introduced three qualitative categories A, B, C, corresponding to the possible drop dynamics scenarios following bubble collapse shown in Fig. 9.

The geometrical quantities dominating the post-collapse dynamics are (1) the drop size, expressed by the minimum drop radius $R_{\mathrm{d} \text { min }}$ before bubble generation, (2) the bubble size, given by the maximum bubble radius $R_{\mathrm{b}, \max }$, and (3) the bubble's radial position within the drop, characterized by the distance $d$ between drop center and bubble center.
Bubble at maximal radius
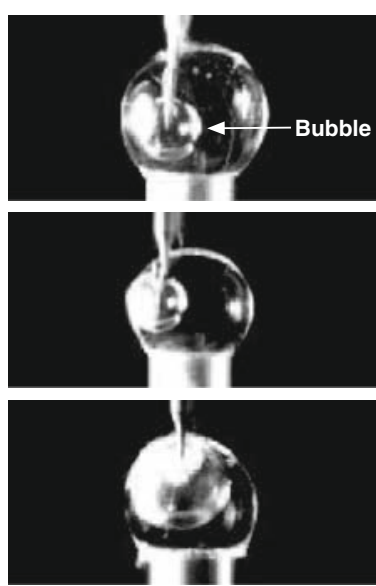

Fig. 9 Three qualitative categories characterizing the strength of jets emission and the stability of the water drop and its surface after bubble collapse. Left column: instant of maximum bubble size, illustrating the relative bubble radius and its position within the drop. Right column: situation $4 \mathrm{~ms}$ after, illustrating the three post-collapse dynamics categories. Cat. A: the microjet escapes but the splash does not. Cat. B: the splash escapes and the microjet is more violent. Cat. $\mathrm{C}$ : the entire drop bursts

But these three parameters are redundant, inasmuch as a uniform stretching of all parameters reproduces a geometrically similar case. A natural choice of non-redundant dimensionless parameters was then obtained by defining: the relative bubble radius $\alpha \equiv R_{\mathrm{b}, \max } / R_{\mathrm{d}, \min }$ and the bubble eccentricity $\varepsilon \equiv d / R_{\mathrm{d}, \min }$.

For the 67 parabolas that qualified for this study (successful bubble generation, quasi-spherical drop, jets within the high-speed camera field of view), the geometrical parameters $\alpha$ and $\varepsilon$ were measured as follows. Because of the drop deviation from spherical symmetry in presence of the electrodes (see Sect. 3.2), the geometrical drop center could not be defined accurately in most parabolas. We therefore adopted the natural convention to measure $R_{\mathrm{d} \text {,min }}$ and $d$ along the axis of jets emission, being the relevant physical axis for the dynamics of the system. As this axis was determined only after the jets emission, it was exported to the first frames of the movies for the measurement of $R_{\mathrm{d} \text {,min }}$ and $d$. To obtain the true position (i.e., corrected for optical refraction through the drop's surface) of the bubble center on this axis, the outer part of the electrode tips were prolongated inside the drop until its intersection with the jets axis. Finally, $R_{\mathrm{b}, \max }$ was obtained from the linear regression between bubble energy and electrostatic energy $E_{\mathrm{b}}\left(E_{\mathrm{e}}\right)$ (derived in Sect. 3), provided the corresponding value of the capacitance (and the electrostatic energy thereof) and the bubble energy-maximum radius relation $E_{\mathrm{b}}\left(R_{\mathrm{b}, \max }\right)$.

We then assigned each of these 67 parabolas to one of the three A-B-C categories, and represented them in the 
dimensionless parameter space $\{\varepsilon, \alpha\}$ shown in Fig. 10. In that space, we can roughly recognize three domains corresponding to the categories $\mathrm{A}, \mathrm{B}$ and $\mathrm{C}$, for which we have drawn straight boundaries to guide the eye (dashed lines). It can be observed that for $0.3<\alpha<0.6$, increasing the eccentricity leads to a transition from category A to category B. A similar transition occurs by increasing $\alpha$ for $0.25<\varepsilon<0.45$. Hence, the splash strength qualitatively increases both with $\alpha$ and $\varepsilon$, as larger values of these parameters may produce a stronger pressure peak between the bubble boundary and the local free surface (as depicted in the numerical simulations of Robinson et al. 2001). Notably, for relative bubble radii above $\alpha \sim 0.6$, we assist to a burst of the entire water drop (cat. C). A closer look at the five cases belonging to this category revealed that the drops remain essentially stable during bubble growth and collapse, but explode immediately after, presumably under the effect of the strong shock wave radiated by the rebounding bubble (Shima 1997).

Consequently, as long as only the cavitation bubble life cycle is to be studied, the whole parameter space $\{\varepsilon, \alpha\}$ can be experimentally explored. However, to investigate the jets dynamics and surface effects, the parameter space should be restricted to $\alpha<0.6$.

\section{Summary and outlook}

We have implemented a simple injector tube system to generate centimetric, quasi-spherical captured drops in microgravity. Mechanical considerations combined with observations revealed that the drop equilibrium is essentially dictated by the ratio of the drop volume to the area of the tube interface. By studying the equilibrium of all the probed configurations, we could estimate the maximum value of this volume-to-contact surface ratio to ensure drop stability.

Single bubbles were generated by spark release between the tips of electrodes immersed within the drop. A careful preparation of the electrode coating was shown to be necessary to avoid drop distortion and deviation from spherical shape. By measuring the maximum bubble radii for various values of the capacitance, we determined the fraction of the electrostatic discharge energy effectively converted into bubble potential energy. In the remaining energy balance, we estimated the fraction of energy carried by the spark shock wave, and lost in electrical Joule heating. Finally, we investigated the influence of the bubble position and size on the strength of the liquid jets and the drop surface perturbations following bubble collapse. It was found that the jets become more prominent as both the

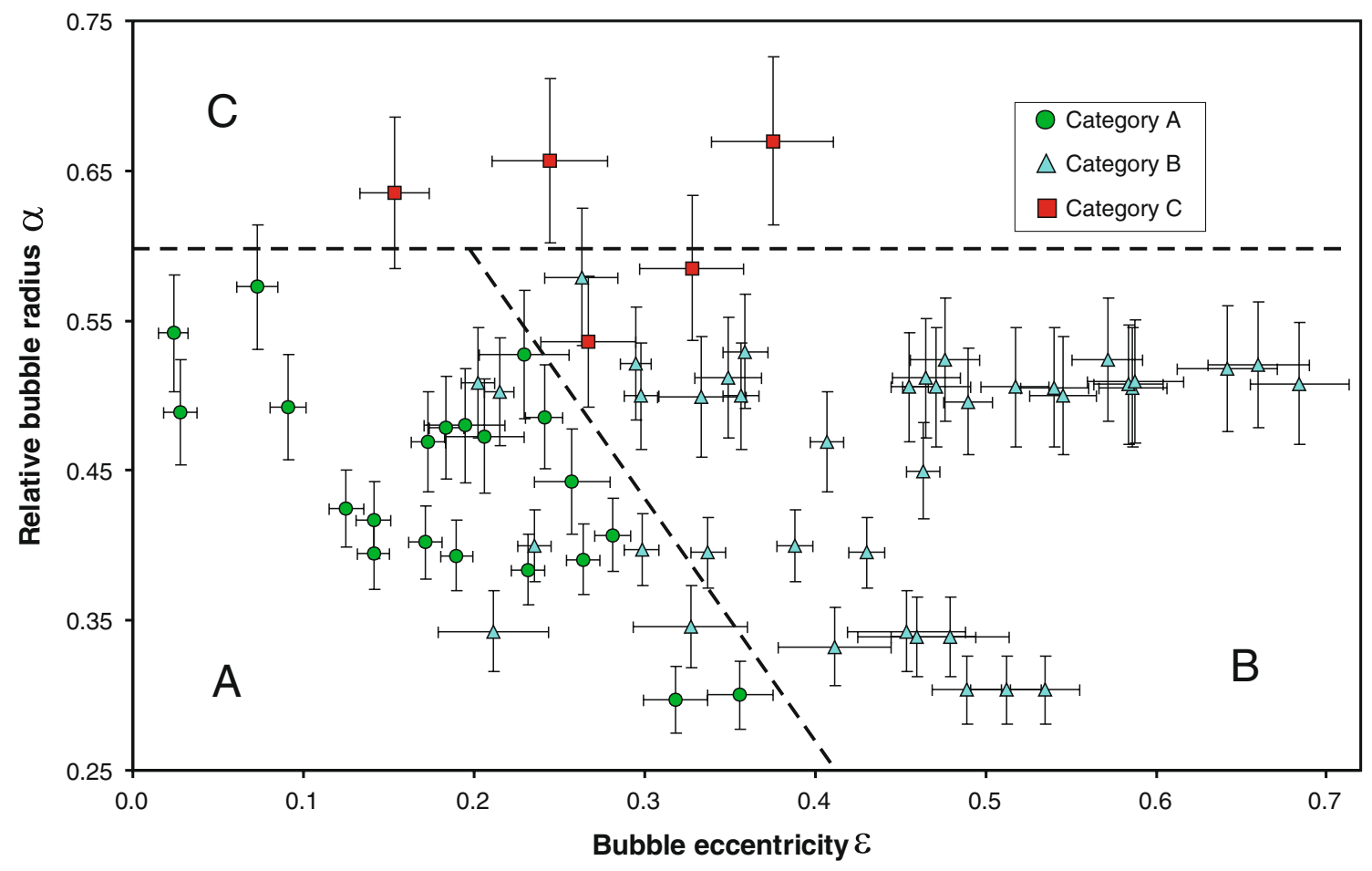

Fig. 10 Representation of 67 parabolas in the parameter space (bubble eccentricity $\varepsilon$, relative bubble radius $\alpha$ ). The parabola points $(\varepsilon, \alpha)$ belonging to different categories A, B, C (introduced in Fig. 9) were plotted with different symbols, in order to identify associated parameter space domains. Straight lines roughly delimiting these domains were overdrawn (dashed). The error bars for $\varepsilon$ depend on the optical resolution (objective focal length), while the uncertainty on $\alpha$ was propagated from the uncertainty on the $E_{\mathrm{b}}\left(E_{\mathrm{e}}\right)$ regression (Sect. 3.3) 
bubble eccentricity and size increase. In particular, for relative bubble radii large enough, the whole drop occurs to burst immediately after bubble collapse.

Cavitation studies apart, our simple and low cost drop generator could offer other interesting applications. In particular, we mention the study of capillary waves on a spherical free surface, as well as the modes and frequencies of a harmonically excited captured drop (numerically simulated by Bauer and Chiba 2004). In the aim of realizing two-phase flows in microgravity conditions, a similar drop generator could be used to study the process of drop formation in flowing liquids, which could also be compared with theoretical models (Kim et al. 1994).

Acknowledgments We gratefully acknowledge the European Space Agency (ESA) for having offered the possibility to pursue these experiments on parabolic flights. Furthermore, our gratitude is directed to the Swiss National Science Foundation (SNSF) and our private donator, the Swiss Space Industry Group (SSIG), who provided the substantial basis of the whole research frame. We also thank several institutions of the Ecole Polytechnique Fédérale de Lausanne (EPFL), the EPFL Space Center in particular, for their support in financial, practical and theoretical aspects. Finally, we adress a special thank to Claude Nicollier, for having kindly offered his time for our support, and provided fruitful advices and experiences.

Open Access This article is distributed under the terms of the Creative Commons Attribution Noncommercial License which permits any noncommercial use, distribution, and reproduction in any medium, provided the original author(s) and source are credited.

\section{Appendix: Optical distortion by a liquid drop}

The image of the bubble inside the water drop is distorted due to optical refraction at the drop's surface. We show that the apparent image is mostly too large, the linear stretching being approximately equal to the index of refraction of water $\eta_{\text {water }}$.

We consider a point located inside a spherical water drop at a distance $r$ from the drop center and placed perpendicularly to the optical axis (Fig. 11). From outside the drop, the point seems to lie at an apparent position $r^{*}$. The geometrical problem may be reduced by considering a two-dimensional drop section parallel to the optical axis, containing both the drop center and the observed point. For simplicity, only the relevant quarter of the drop section is sketched here. From Fig. 11 we derive the geometrical relation

$r^{*}-r=\sqrt{R_{\mathrm{d}}^{2}-r^{* 2}} \tan (\alpha)=\sqrt{R_{\mathrm{d}}^{2}-r^{* 2}} \tan \left(\beta^{*}-\beta\right)$

where $\sin \left(\beta^{*}\right)=r^{*} / R_{\mathrm{d}}$. To eliminate $\beta$, Snell's law of refraction is applied,

$\sin (\beta)=\frac{1}{\eta_{\text {water }}} \sin \left(\beta^{*}\right)=\frac{r^{*}}{\eta_{\text {water }} R_{\mathrm{d}}}$

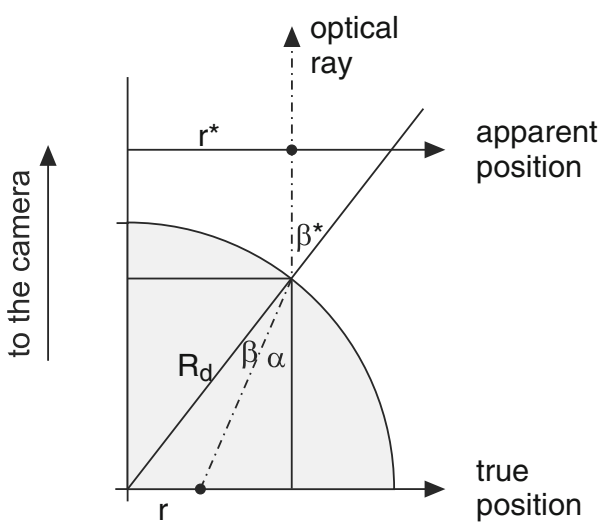

Fig. 11 Model of optical refraction by a spherical drop. Gray zone: quarter of the drop's cross-section. Dashed-dot line: optical ray

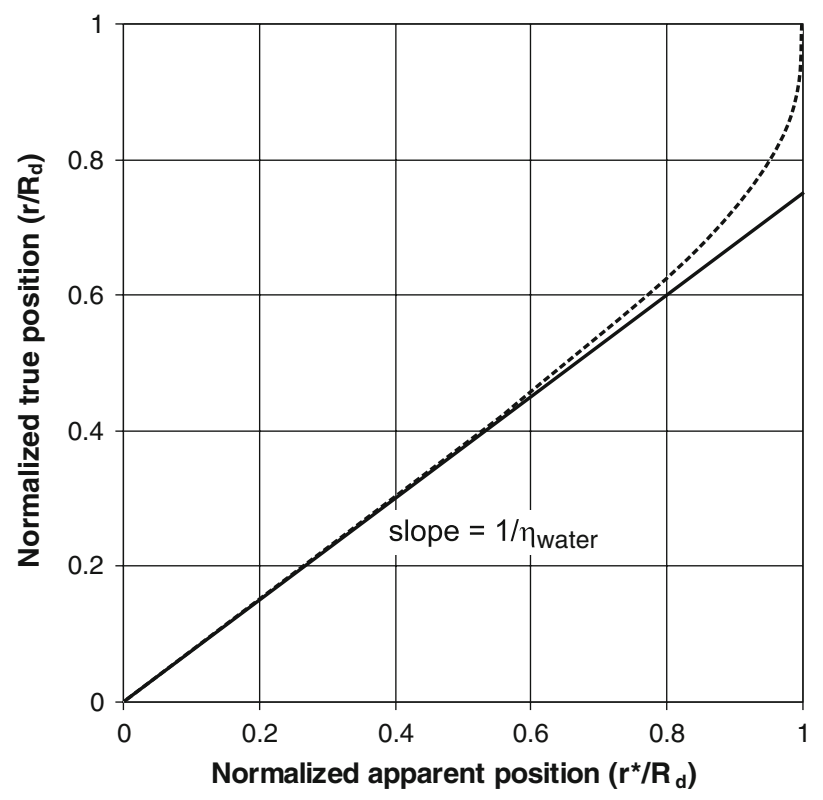

Fig. 12 Solid line: true position versus apparent position of a point inside a water sphere, observed perpendicularly to the straight line connecting it to the spheres center. Dashed line linear expansion about $r=r^{*}=0$

After substitution and rearrangement, the following relation between $r$ and $r^{*}$ is obtained,

$r=r^{*}-\sqrt{R_{\mathrm{d}}^{2}-r^{* 2}} \times \tan \left(\arcsin \frac{r^{*}}{R_{\mathrm{d}}}-\arcsin \frac{r^{*}}{\eta_{\mathrm{water}} R_{\mathrm{d}}}\right)$

Figure 12 (solid line) shows $r / R_{\mathrm{d}}$ in function of $r^{*} / R_{\mathrm{d}}$ for the particular index $\eta_{\text {water }}=1.33$. Over the wide range $r^{* /}$ $R_{\mathrm{d}}<0.6$ this function is nearly linear obeying the simple law (dashed line),

$r^{*}=\eta_{\text {water }} r \quad\left(\right.$ linear expansion about $\left.r=r^{*}=0\right)$ 


\section{References}

Apfel RE, Tian Y, Jankovsky J, Shi T, Chen X, Holt RG, Trinh E, Croonquist A, Thornton K, Sacco AJ, Coleman C, Leslie FW, Matthiesen DH (1997) Free oscillations and surfactant studies of superdeformed drops in microgravity. Phys Rev Lett 78:19121915

Bauer HF, Chiba M (2004) Oscillations of captured spherical drop of frictionless liquid. J Sound Vib 274:725-746

Blake JR, Gibson DC (1981) Growth and collapse of a vapour cavity near a free surface. J Fluid Mech 111:123-140

Brennen CE (1995) Cavitation and bubble dynamics. Oxford University Press, New York

Brenner MP, Hilgenfeldt S, Lohse D (2002) Single-bubble sonoluminescence. Rev Mod Phys 74:425-484

Brujan EA, Keen GS, Vogel A, Blake JR (2002) The final stage of the collapse of a cavitation bubble close to a rigid boundary. Phys Fluids 14:85-92

Chahine GL, Frederik GS, Lambrecht CJ, Harris GS, Mair HU (1995) Spark-generated bubbles as laboratory-scale models of underwater explosions and their use for validation of simulation tools. In: SAVIAC proceedings 66th shock and vibrations symposium, Biloxi, vol 2, pp 265-276

Chen X, Shi T, Tian Y, Jankovsky J, Holt R, Apfel R (1998) Numerical simulation of superoscillations of a triton-bearing drop in microgravity. J Fluid Mech 367:205-220

Croonquist AP (1993) The drop physics module on USML-1 DPM hardware and science. In: AIAA, space programs and technologies conference and exhibit, Huntsville, no. 4309 in AIAA papers

Faraday M (1831) On a peculiar class of acoustical figures and on certain forms assumed by groups of particles upon vibrating elastic surfaces. Philos Trans R Soc Lond 121:299-340

Holt R, Trinh EH (1996) Faraday wave turbulence on a spherical liquid shell. Phys Rev Lett 77:1274-1277
Holt R, Tian Y, Jankovsky J, Apfel R (1997) Surface-controlled drop oscillations in space. J Acoust Soc Am 102:3802-3805

Isselin JC, Alloncle AP, Autric M (1998) On laser induced single bubble near a solid boundary: contribution to the understanding of erosion phenomena. J Appl Phys 84:5766-5771

Kim I, Kamotani Y, Ostrach S (1994) Modeling bubble and drop formation in flowing liquids in microgravity. AIChE $\mathbf{J} 40: 19-28$

Leighton TG (1994) The acoustic bubble. Academic Press, London

Lord Rayleigh (1879) On the capillary phenomena of jets. Proc Roy Soc Lond 29:71-97

Obreschkow D, Kobel P, Dorsaz N, de Bosset A, Nicollier C, Farhat M (2006) Cavitation bubble dynamics inside liquid drops in microgravity. Phys Rev Lett 97:094502-1-4

Pearson A, Cox E, Blake JR, Otto SR (2004) Bubble interactions near a free surface. Eng Anal Bound Elem 28:295-313

Pereira F, Farhat M, Avellan F (1994) Dynamic calibration of transient sensors by spark generated cavity. In: Blake JR et al (ed) Bubble dynamics and interface phenomena. Kluwer, Dordrecht, pp 227-240

Robinson RB, Blake JR, Kodama T, Shima A, Tomita Y (2001) Interaction of cavitation bubbles with a free surface. J Appl Phys 89:8225-8237

Shima A (1997) Studies on bubble dynamics. Shock Waves 7:33-42

Tomita Y, Robinson RB, Tong RP, Blake JR (2002) Growth and collapse of cavitation bubbles near a curved rigid boundary. J Fluid Mech 466:259-283

Treuner M, Rath HJ, Duda U, Siekman J (1995) Thermocapillary flow in drops under low gravity analysed by the use of liquid crystals. Exp Fluids 19:264-273

Wang TG, Trinh EH, Croonquist AP, Elleman DD (1986) Shapes of rotating free drops: spacelab experimental results. Phys Rev Lett $56: 452-455$

Young T (1845) A course of lectures on natural philosophy and the mechanical arts. Taylor and Walton, London 